\title{
BOLD fMRI activation induced by vagus nerve stimulation in seizure patients
}

\author{
W-C Liu, K Mosier, A J Kalnin, D Marks
}

J Neurol Neurosurg Psychiatry 2003;74:81 1-813

Objective: To identify the cerebral activated regions associated with the vagus nerve stimulation in epilepsy patients.

Design: Blood oxygenation level dependent functional magnetic resonance imaging (BOLD FMRI) was employed to detect areas of the brain activated by vagus nerve stimulation in five patients with documented complex partial seizures.

Methods: Functional MRI was done on a GE 1.5T Echospeed horizon scanner. Before each patient entered the scanner, the vagal nerve stimulator was set to a specific ON-OFF paradigm so that the data could be analysed using a box-car type of design. The brains were scanned both anatomically and functionally. The functional images were corrected for head motion and co-registered to the anatomical images. Maps of the activated areas were generated and analysed using the brain mapping software, SPM99. The threshold for activation was chosen as $p<0.001$.

Results: All patients showed activation in the frontal and occipital lobes. However, activation in the thalamus was seen only in the two patients with improved seizure control. Conclusions: BOLD FMRI can detect activation associated with vagus nerve stimulation. There may be a relation between thalamic activation and a favourable clinical outcome.

$\mathrm{V}$ agus nerve stimulation has been used to treat patients with complex partial seizures. This is achieved using an implantable nerve stimulator (NeuroCybernetic prothesis or NCP system, Cyberonics Inc, Webster, Texas, USA). ${ }^{1}$ Currently, more than 2000 patients with refractory epilepsy are treated each year with this device. ${ }^{2}$ The mechanisms underlying this treatment are still unclear.

A PET study ${ }^{3}$ reported that patients with increased thalamic blood flow showed improvement in seizure control in response to vagus nerve stimulation. Vagus nerve stimulation of the thalamus may play an important role in controlling seizures.

In addition to vagus nerve stimulation, studies using direct electrical stimulation of the anterior ${ }^{4}$ or centromedian ${ }^{5}$ thalamus were reported to be potential treatments for refractory epilepsy.

Recently, brain activation by vagus nerve stimulation was detected using blood oxygenation level dependent functional magnetic resonance imaging (BOLD fMRI). ${ }^{6}$ BOLD fMRI is a non-invasive technique that uses local changes in the concentrations of oxyhaemoglobin and deoxyhaemoglobin to identify regions of increased neural activity. ${ }^{7}$ Our aim in the present study was to identify cerebral regions activated during vagus nerve stimulation in a population with complex partial seizures. This information may be helpful in understanding the therapeutic mechanisms of vagus nerve stimulation.

\section{METHODS \\ Patients}

Five patients with documented complex partial seizures (four men, one woman; mean age 32 years) had an NCP device implanted at least one month before enrolment in the study (table 1). For each patient we calculated two seizure frequencies (from the patient's seizure diary): a preimplantation frequency for the preceding two months, and a postimplantation frequency after eight to 10 months. Anticonvulsants were not changed following NCP implantation and the patients were followed up to 16 months postimplantation.

Before the imaging, all patients signed a consent form approved by the institutional review board of our institution. On the day of scanning, they were examined by the neurologist (DM). When the stimulator was activated, all patients

Abbreviations: BOLD FMRI, blood oxygenation level dependent magnetic resonance imaging; FOV, field of view; MNI, Montreal Neurological Institute; NCP, NeuroCybernetic prothesis; TE, time of echo; $T R$, time of repetition

Table 1 Demographic and thalamic activation data during vagus nerve stimulation

\begin{tabular}{|c|c|c|c|c|c|}
\hline \multirow[b]{2}{*}{$\begin{array}{l}\text { Patient No/age (years)/sex/seizure } \\
\text { focus/anticonvulsant }\end{array}$} & \multirow[b]{2}{*}{$\begin{array}{l}\text { NCP implanted } \\
\text { before scanning }\end{array}$} & \multirow[b]{2}{*}{ Frequency* } & \multirow[b]{2}{*}{$\begin{array}{l}\text { Clinical improvement } \\
(\%)\end{array}$} & \multicolumn{2}{|c|}{ Thalamic activation during VNS } \\
\hline & & & & $\begin{array}{l}\text { Cluster extent } \\
\text { (voxel) }\end{array}$ & $\mathrm{p}$ Value \\
\hline 1/27/M/BTL/topiramate, valproic acid & 1 month & 10 & $0 \%$ & 0 & \\
\hline $2 / 29 / F / R F T L / c a r b a m a z e p i n e$ & 2 months & 0.7 & $+75 \%$ & 22 & $p<2.7 \times 10^{-4}$ \\
\hline 3/26/M/RTL/carbamazepine & 11 months & 4 & $0 \%$ & 0 & \\
\hline 4/40/M/RTL/topiramate, valproic acid & 6 months & 4 & $0 \%$ & 0 & \\
\hline $5 / 40 / \mathrm{M} / \mathrm{LTL} /$ topiramate, valproic acid & 5 months & 1 & $+50 \%$ & 5 & $p<3.6 \times 10^{-6}$ \\
\hline
\end{tabular}

*Number of seizures/month on average before the implantation.

BTL, bilateral temporal lobes; F, female; LTL: left temporal lobe; $M$, male; RFTL, right frontal temporal lobe; RTL, right temporal lobe; VNS, vagus nerve stimulation. 

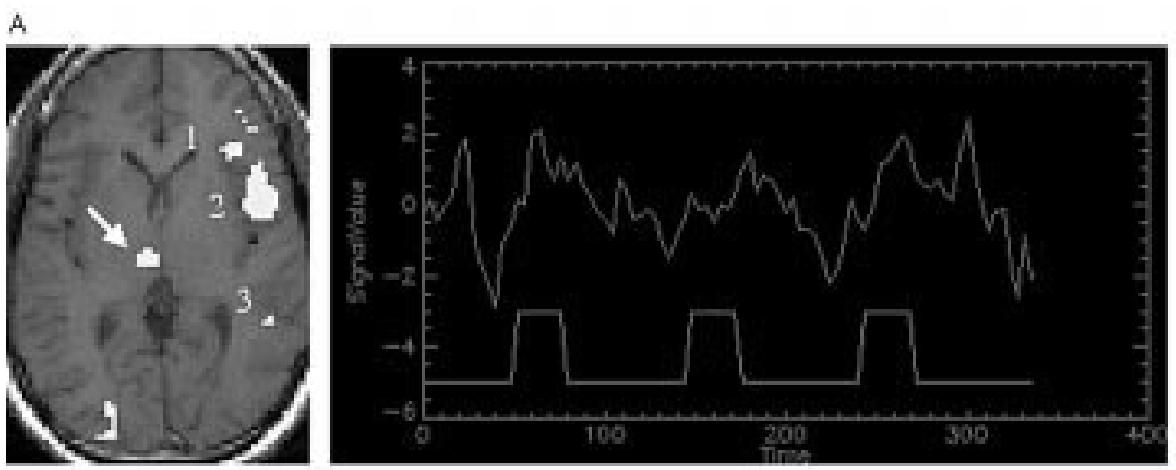

B
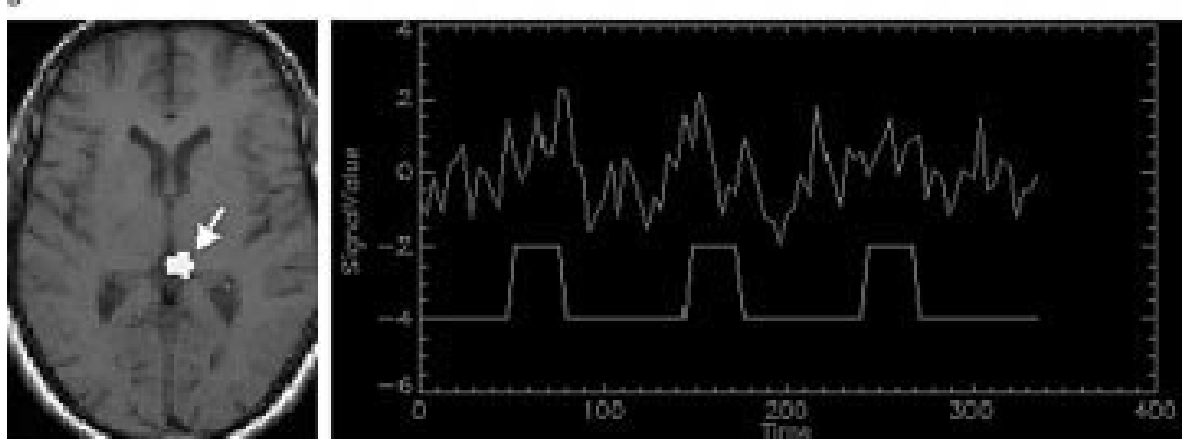

Figure 1 Thalamic activation and its time course during vagus nerve stimulation from two patients who showed clinical improvement during the study. (A) Brain map of thalamic activation and its time course from patient No 2. The cerebral activation was observed from thalamus (arrow), insula (1), frontal lobe (2), and temporal lobe (3). The plot shows the time course of activation in the thalamus (upper curve) and the NCP stimulation paradigm (lower curve) during vagus nerve stimulation. (B) Thalamic activation (arrow) and the time course of activation from patient No 5 .

reported a tingling sensation over the left cervical region, throat irritation, and change in pitch when speaking.

\section{Experimental design}

For fMRI data acquisition, the NCP stimulation parameters were set by an external magnetic wand to a pattern of 30 seconds ON and 66 seconds OFF; all other parameters of the device-that is, signal frequency $(30 \mathrm{~Hz})$, pulse width $(250$ $\mu \mathrm{s})$, and output current ( 1.25 to $1.75 \mathrm{~mA}$ )-remained the same. All patients wore a $30 \mathrm{~dB}$ noise reduction headset to reduce loud noise ( $>100 \mathrm{~dB}$ ) generated by the magnetic resonance scanner; this also supported the head in a more stable position. Before scanning, patients were re-evaluated to determine whether they could reliably report the ON/OFF status of the stimulator

\section{Functional $M R$ imaging}

Imaging was done with a GE 1.5T Echospeed horizon scanner. Before the functional MRI, a set of high resolution Tl weighted co-planar images was obtained for overlay on the functional datasets. The parameters for the $\mathrm{Tl}$ weighted images were TR/TE $=550 / \mathrm{min}, \mathrm{FOV}=24 \mathrm{~cm}, 256 \times 256$ matrix, 28 slices with $5 \mathrm{~mm}$ slice thickness. For the functional images the scanning parameters were $\mathrm{TR} / \mathrm{TE}=4000 / 60 \mathrm{~ms}$, FOV $=24 \mathrm{~cm}$, matrix size $=64 \times 64$, and 28 slices continuously at $5 \mathrm{~mm}$ slice thickness.

Functional imaging was begun 30 seconds after the patient reported that the stimulator was ON. For each patient, three consecutive scans were acquired with a 30 second pause between each scan. Each scan lasted five minutes and 56 seconds and consisted of a baseline of 66 seconds (stimulator OFF) followed by three cycles of 30 seconds ON and 66 seconds OFF.

At the conclusion of the study, the patient's stimulator was reset to the original parameters.

\section{Data analysis}

For each patient the three scans were reconstructed separately and a final brain map was generated from the average of the scans. The functional data were reconstructed off-line on a Sun Ultra60 workstation and processed and analysed using the brain mapping software, SPM99. ${ }^{8}$ For each scan, the reconstructed images were realigned, co-registered to the anatomical Tl images, normalised to Montreal Neurological Institute (MNI) standard brain, and then converted to Talairach brain space. Head motion artefact was accounted for by treating head displacement as a covariate. A final map for each patient was then generated by averaging the data from the three consecutive scans. The anatomical locations corresponding to the activated regions $(p<0.001)$ were identified using available digital mapping software ${ }^{9}$ and confirmed by the neuroradiologist (AJK).

\section{RESULTS}

Areas of activation were seen in frontal and occipital lobes (in all five patients), parietal lobe (in four patients), middle temporal lobe and cingulate gyrus (in three patients), and insula and thalamus (in two patients; fig 1). In addition, activation of the cerebellum was observed in four patients. The detailed activation areas are listed in table 2 .

Both patients with clinical improvement (Nos 2 and 5) had thalamic activation. Patient No 2, with 75\% improvement, had a reduction in the number of seizures from one every one to two months ( 0.7 seizure/month) to one in six months $(0.2$ seizure/month). Patient No 5, with a 50\% improvement, had a decrease in seizure frequency from one seizure a month before the treatment to one every two to three months (0.4 seizure/month) following implantation of the stimulator. The remaining patients showed no change in seizure frequency (table 1). 
Table 2 The activated regions observed in the left and right hemispheres for all five patients

\begin{tabular}{|c|c|c|c|c|c|c|c|c|c|c|}
\hline & \multicolumn{2}{|c|}{ Patient 1} & \multicolumn{2}{|c|}{ Patient 2} & \multicolumn{2}{|c|}{ Patient 3} & \multicolumn{2}{|c|}{ Patient 4} & \multicolumn{2}{|c|}{ Patient 5} \\
\hline & Left & Right & Left & Right & Left & Right & Left & Right & Left & Right \\
\hline Cereb & 3 & & & & 50 & 14 & 28 & 72 & 160 & 251 \\
\hline IFG & & 3 & & 28 & 8 & 4 & & 4 & & \\
\hline MFG & & & & & & 7 & & & 6 & 30 \\
\hline SFG & & & 6 & 7 & & & & 3 & 3 & 9 \\
\hline MTG & & 6 & & 15 & & & & 17 & & \\
\hline STG & 4 & & & 32 & 7 & 4 & 7 & & 6 & 14 \\
\hline SUPMG & & & & & & 12 & & & 7 & \\
\hline SPG & & 3 & 15 & 9 & & & & 9 & 9 & 19 \\
\hline$A n G$ & & & & 10 & & 19 & 3 & & & 11 \\
\hline Occipital & & 8 & 8 & 7 & & 4 & 23 & 10 & 8 & 11 \\
\hline Insula & & & & 14 & & & 9 & & & \\
\hline Cingulate & & 5 & 13 & 37 & & 17 & & & & \\
\hline Thalamus & & & 9 & 11 & & & & & & 5 \\
\hline
\end{tabular}

The values are the number of voxels (exceeding an extent threshold of 2 voxels and height threshold of $p<0.001$ ) for each region. The voxel has a resampled unit of $2 \mathrm{~mm}^{3}$.

AnG, angular gyrus; cereb, cerebellum; cingulate, cingulate gyrus; IFG, inferior frontal gyrus; insula, insular cortex; MFG, middle frontal gyrus; MTG, middle temporal gyrus; occipital, occipital lobe; SFG, superior frontal gyrus; SPG, superior parietal gyrus; STG, superior temporal gyrus; SUPMG, supramarginal gyrus.

\section{DISCUSSION}

In our study, activation was detected in the frontal and occipital lobes in all patients. This finding has been reported in other fMRI studies examining the effects of vagus nerve stimulation. $^{3}$ Anatomically, ascending pathways ${ }^{2}$ from the vagus nerve in the nucleus of the solitary tract project to the parabrachial nucleus. The parabrachial nucleus projects to several forebrain areas: to the hypothalamus, amygdala, and the infralimbic cortex; to the ventroposterior thalamic complex and insular cortex; and to thalamic intralaminar and middle nuclei and the cerebral cortex. In this study, the activation we observed in frontal lobes, insula, and cingulate gyrus is consistent with the known terminal connections of the parabrachial nucleus and the thalamus. Thus activation measured by fMRI during vagus nerve stimulation appears to correspond to regions involved in the cortical processing of vagal inputs.

Although our sampling numbers are small, the two patients with thalamic activation had a more than $50 \%$ seizure reduction after implantation. Of these two patients, the one with greater seizure control had a more robust thalamic activation pattern. Thus the brain maps seemed to show a good correlation of thalamic activation with clinical improvement. This finding is in agreement with the results of a study using PET. ${ }^{3}$

The thalamocortical system is involved in generating both physiological and pathological rhythms, including sleep spindle waves and spike-wave discharges. Modulation of the thalamocortical networks by vagus nerve stimulation may influence seizure control by disrupting these neural networks. ${ }^{10}$ Our study suggests that significant thalamic activation in both spatial extent (cluster size) as well as peak intensity ( $p$ value) may have important clinical implications.

\section{ACKNOWLEDGEMENTS}

We would like to thank Dr Joseph Maldjian (Wake Forest University, School of Medicine) for software support and Dr Susan C Feldman (New Jersey Medical School) for scientific input to the manuscript. This work was supported by a seed grant from Cyberonics Inc.

\section{Authors' affiliations}

W-C Liu, K Mosier, A J Kalnin, Department of Radiology, University of Medicine and Dentistry of New Jersey, New Jersey Medical School, Newark, New Jersey, USA

D Marks, Department of NeuroSciences, University of Medicine and Dentistry of New Jersey

Competing interests: This work was supported by a seed grant from Cyberonics Inc, Webster, Texas, USA

Correspondence to: Dr Wen-Ching Liu, Department of Radiology, University of Medicine and Dentistry of New Jersey, New Jersey Medical School, 150 Bergen Street, Newark, NJ 07103, USA; wliu@umdni.edu

Received 21 October 2002

In revised form 18 December 2002

Accepted 17 January 2002

\section{REFERENCES}

1 Terry RS, Tarver WB, Zabara J. The implantable neurocybernetic prosthesis system. Pacing Clin Electrophysiol 1991;14:86-93.

2 Schachter SC, Saper CB. Vagus nerve stimulation. Epilepsia 1998;39:677-86.

3 Henry TR, Votaw JR, Pennell PB, et al. Acute blood flow changes and efficacy of vagus nerve stimulation in partial epilepsy. Neurology 1999:52:1162-73

4 Cooper IS, Upton AR, Amin I, et al. Evoked metabolic responses in the limbic-striate system produced by stimulation of anterior thalamic nucleus in man. Int J Neurol 1984;18:179-87.

5 Velasco $F$, Velasco M, Ogarrio C, et al. Electrical stimulation of the centromedian thalamic nucleus in the treatment of convulsive seizures: a preliminary report. Epilepsia 1987;28:421-30.

6 Bohning DE, Lomarev MP, Denslow S, et al. Feasibility of vagus nerve stimulation-synchronized blood oxygenation level-dependent functional MRI. Invest Radiol 2001:36:470-9.

7 Ogawa S, Lee T, Kay AR, et al. Brain magnetic resonance imaging with contrast dependent on blood oxygenation. Proc Natl Acad Sci USA 1990;87:9868-72.

8 Friston KJ, Holmes AP, Worsley KJ, et al. Statistical parametric maps in functional imaging: a general linear approach. Hum Brain Map 1995; 2:189-210.

9 Kikinis R, Shenton M, Losifescu D, et al. A digital brain atlas for surgical planning, model driven segmentation and teaching. IEEE Trans Visual Comput Graphics 1996:2:2223-41.

10 Engel J, Pedley TA. Epilepsy: a comprehensive textbook. Philadelphia: Lippincott-Raven, 1997. 